TAUP 2125-93

hep-th/9312043

\title{
The Many Faces of a Character
}

\author{
Ezer Melzer \\ School of Physics and Astronomy \\ Beverly and Raymond Sackler Faculty of Exact Sciences \\ Tel-Aviv University \\ Tel-Aviv 69978, ISRAEL \\ email: melzer@ccsg.tau.ac.il
}

\begin{abstract}
We prove an identity between three infinite families of polynomials which are defined in terms of 'bosonic', 'fermionic', and 'one-dimensional configuration' sums. In the limit where the polynomials become infinite series, they give different-looking expressions for the characters of the two integrable representations of the affine $s u(2)$ algebra at level one. We conjecture yet another fermionic sum representation for the polynomials which is constructed directly from the Bethe-Ansatz solution of the Heisenberg spin chain.
\end{abstract}




\section{Introduction}

General symmetry arguments lead to the observation that certain infinite-dimensional algebras, called chiral or vertex operator algebras, play a major role in two-dimensional conformal field theory [1] [2]. In particular, the Hilbert space of a conformal field theory decomposes into irreducible highest-weight representations of such algebras. The theory of such representations is well developed [3], and their characters, which encode the spectrum of (certain sectors of) the conformal field theory, have been calculated extensively by various methods. The characters are constructed as formal power series $\chi(q)$ in some variable $q$ (occasionally other variables - "counting" the charges with respect to some symmetry - are also present).

Such characters also arise in studies of integrable models of two-dimensional classical statistical mechanics and their related (through a transfer matrix) one-dimensional quantum systems. This comes as no surprise when the two- (one-)dimensional system involved is critical (gapless), as - having in mind the discussion above - scaling limits of many such systems are expected to be described by conformal field theories. More surprising is the appearance of characters in computations of order parameters in off-critical two-dimensional systems, using Baxter's corner transfer matrix (CTM) technique [⿶凵⿴囗十

An interesting aspect for us here is the possibility, due to the fact that the statistical mechanics models are defined as finite systems of size $L=1,2,3, \ldots$, to construct infinite families of polynomials $\chi^{(L)}(q)$ which approach the characters in the thermodynamic limit $L \rightarrow \infty$. In a sense, this procedure describes a gradual build up of the infinite representation space of the relevant chiral algebra. Now, within different frameworks one obtains different-looking expressions for the finitized characters $\chi^{(L)}(q)$. Analyses [5] of the spectrum of certain gapless spin-chain hamiltonians using Bethe-Ansatz-type methods suggests that such methods generically lead to fermionic sum expressions [6]-[8] for the (finitized) characters, expressions which generalize the (finitized) sum-side of the Rogers-RamanujanSchur identities; CTM methods, on the other hand, lead naturally to one-dimensional (1D) configuration sums, which are statistical sums over certain restricted configurations (with appropriate weights) defined on a finite one-dimensional lattice.

In [9] we noted the equality of certain fermionic and $1 \mathrm{D}$ configuration sums, which are finitized characters of the unitary minimal models of conformal field theory. These 1D configuration sums appeared originally in the work [10] on RSOS models, and were shown there to be equal to certain bosonic sums which provide finitizations of the more familiar expressions for the characters [11] given in terms of theta functions. 
In the present paper we discuss analogous identities between bosonic, fermionic, and 1D configuration sum expressions for finitized characters of the $s u(2)$ affine Kac-Moody algebra at level one. The relevant spin chain in this case is [12] the (antiferromagnetic) spin- $\frac{1}{2}$ Heisenberg chain, to which the Bethe Ansatz was originally applied [13]. We will see that the Bethe-Ansatz description of its spectrum leads naturally to a decomposition of the affine $s u(2)$ characters into characters of the so-called degenerate representations of the Virasoro algebra at central charge $c=1$, thus motivating us to conjecture fermionic sum expressions also for finitizations of the latter characters.

\section{Polynomial Identities}

We start by recalling some standard notation (see e.g. 14]). Let

$$
(q)_{0}=1 \quad, \quad(q)_{m}=\prod_{\ell=1}^{m}\left(1-q^{\ell}\right) \quad \text { for } m=1,2,3, \ldots
$$

in terms of which the $q$-binomial coefficients are defined as

$$
\left[\begin{array}{l}
n \\
m
\end{array}\right]_{q}= \begin{cases}\frac{(q)_{n}}{(q)_{m}(q)_{n-m}} & \text { if } 0 \leq m \leq n \\
0 & \text { otherwise }\end{cases}
$$

for $m, n \in \mathbb{Z}$. Note their properties [14]

$$
\begin{gathered}
{\left[\begin{array}{l}
n \\
m
\end{array}\right]_{q}=\left[\begin{array}{c}
n-1 \\
m-1
\end{array}\right]_{q}+q^{m}\left[\begin{array}{c}
n-1 \\
m
\end{array}\right]_{q}} \\
=\left[\begin{array}{c}
n-1 \\
m
\end{array}\right]_{q}+q^{n-m}\left[\begin{array}{c}
n-1 \\
m-1
\end{array}\right]_{q}, \\
{\left[\begin{array}{c}
n_{1}+n_{2} \\
m^{\prime}
\end{array}\right]_{q}=\sum_{m_{2} \in \mathbb{Z}} q^{m_{2}\left(m_{2}+m^{\prime}-n_{2}\right)}\left[\begin{array}{c}
n_{1} \\
m_{2}+m^{\prime}-n_{2}
\end{array}\right]_{q}\left[\begin{array}{c}
n_{2} \\
m_{2}
\end{array}\right]_{q} \quad\left(n_{1}, n_{2} \geq 0\right),} \\
\lim _{n \rightarrow \infty}\left[\begin{array}{c}
n \\
m
\end{array}\right]_{q}=\frac{1}{(q)_{m}} \quad(m \geq 0), \quad \lim _{n, m \rightarrow \infty}\left[\begin{array}{c}
n+m \\
m
\end{array}\right]_{q}=\frac{1}{(q)_{\infty}}, \\
\lim _{q \rightarrow 1}\left[\begin{array}{c}
n \\
m
\end{array}\right]_{q}=\left(\begin{array}{c}
n \\
m
\end{array}\right)=\frac{n !}{m !(n-m) !} \quad(0 \leq m \leq n) .
\end{gathered}
$$

Now for $j=0, \frac{1}{2}$ and $L$ a non-negative integer, define the bosonic sums

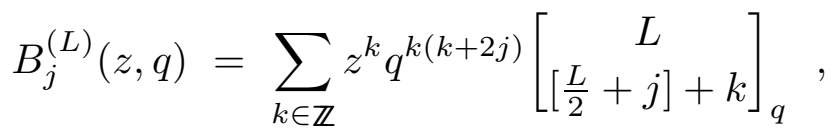


where $[x]$ denotes the integer part of $x$. Also, define the fermionic sums

$$
F_{j}^{(L)}(z, q)=\sum_{m_{1}, m_{2} \in \mathbb{Z}} z^{m_{1}-m_{2}} q^{\frac{1}{2} \mathbf{m} C_{A_{2}} \mathbf{m}^{t}+2 j\left(m_{1}-m_{2}\right)}\left[\begin{array}{c}
\left.\frac{L+1}{2}-j\right] \\
m_{1}
\end{array}\right]_{q}\left[\left[\begin{array}{c}
\left.\frac{L}{2}+j\right] \\
m_{2}
\end{array}\right]_{q}\right.
$$

where $C_{A_{2}}=\left(\begin{array}{cc}2 & -1 \\ -1 & 2\end{array}\right)$ is the Cartan matrix of the algebra $A_{2}$, and $\mathbf{m}=\left(m_{1}, m_{2}\right)$. Finally, let $\mu_{\ell}=\frac{1}{2}\left(1-(-1)^{\ell}\right)$, i.e. $\left(\mu_{1}, \mu_{2}, \mu_{3}, \ldots\right)=(1,0,1, \ldots)$, and for positive $L$ (still $\left.j=0, \frac{1}{2}\right)$ define the $1 \mathrm{D}$ configuration sums

$$
\begin{aligned}
& C_{j}^{(L)}(z, q)=\left(z^{2} q\right)^{2 j(-1)^{L}\left[\frac{L+1}{2}\right]} \sum_{h_{1}, \ldots, h_{L} \in\{0,1\}} z^{\sum_{\ell=1}^{L} \ell\left(\left(h_{\ell+1}-h_{\ell}\right)-\left(\mu_{\ell+1}-\mu_{\ell}\right)\right)} \\
& \times q^{\sum_{\ell=1}^{L} \ell\left(\max \left(1-h_{\ell}, h_{\ell+1}\right)-\max \left(1-\mu_{\ell}, \mu_{\ell+1}\right)\right)}
\end{aligned}
$$

where $h_{L+1}=\mu_{L+1}$ for $j=0$ and $h_{L+1}=1-\mu_{L+1}$ for $j=\frac{1}{2}$. More explicitly, since $\max \left(1-h_{1}, h_{2}\right)=1-h_{1}+h_{1} h_{2}$ for $h_{1}, h_{2} \in\{0,1\}$, we have

$$
\begin{aligned}
C_{j}^{(L)}(z, q)=\left(z^{2} q\right)^{2 j(-1)^{L}\left[\frac{L+1}{2}\right]} \sum_{\substack{h_{1}, \ldots, h_{L} \in\{0,1\} \\
h_{L+1}=\left(1+(-1)^{L+2 j}\right) / 2}} z^{\sum_{\ell=1}^{L} \ell\left(h_{\ell+1}-h_{\ell}-(-1)^{\ell}\right)} \\
\times q^{\sum_{\ell=1}^{L} \ell\left(\frac{1}{2}\left(1-(-1)^{\ell}\right)-h_{\ell}\left(1-h_{\ell+1}\right)\right)},
\end{aligned}
$$

and in addition, for $L=0$, we set $C_{j}^{(0)}(z, q)=1$.

The objects $B_{j}^{(L)}(z, q), F_{j}^{(L)}(z, q)$, and $C_{j}^{(L)}(z, q)$ are polynomials in $q, z$ and $z^{-1}$ whose constant term is 1 , i.e. they can be expanded as finite (for finite $L$ ) sums of the form $\sum_{n=0}^{n_{1}} \sum_{m=m_{0}}^{m_{1}} a_{n m} z^{m} q^{n}$ with $m_{0}$ a (generically) negative integer and $a_{00}=1$ for all $L$ and both values of $j$. For $B_{j}^{(L)}(z, q)$ and $F_{j}^{(L)}(z, q)$ this fact is clear from the definitions, while for $C_{j}^{(L)}(z, q)$ it is best seen from the following theorem which is one of our main results.

Theorem: For $j=0, \frac{1}{2}$ and $L=0,1,2, \ldots$

$$
B_{j}^{(L)}(z, q)=F_{j}^{(L)}(z, q)=C_{j}^{(L)}(z, q) .
$$

Proof: (i) The first equality in (2.11) is proven directly by using the general identity (2.4) specialized to the case of $n_{1}=\left[\frac{L+1}{2}-j\right], n_{2}=\left[\frac{L}{2}+j\right] \quad$ (so that $n_{1}+n_{2}=L$ for both $\left.j=0, \frac{1}{2}\right)$, and $m^{\prime}=\left[\frac{L}{2}+j\right]+k$. The rhs of (2.7) is then rewritten as

$$
B_{j}^{(L)}(z, q)=\sum_{k, m_{2} \in \mathbb{Z}} z^{k} q^{k(k+2 j)+m_{2}\left(m_{2}+k\right)}\left[\begin{array}{c}
\left.\frac{L+1}{2}-j\right] \\
m_{2}+k
\end{array}\right]_{q}\left[\begin{array}{c}
{\left[\frac{L}{2}+j\right]} \\
m_{2}
\end{array}\right]_{q},
$$


and the change of summation variable $k \mapsto m_{1}=k+m_{2}$ gives the rhs of (2.8), as claimed. (ii) To prove the second equality in (2.11) we show that the $B_{j}^{(L)}$ and $C_{j}^{(L)}$ satisfy the same recursion relations and initial conditions in $L$. Starting from the rhs of (2.7), we use the first (second) line of (2.3), followed by a change of summation variable $k \mapsto k^{\prime}=k+2 j$ $\left(k \mapsto k^{\prime}=k-1+2 j\right)$ when $\frac{L}{2}+j \in \mathbb{Z} \quad\left(\frac{L+1}{2}+j \in \mathbb{Z}\right)$ in the second resulting sum. Thus we obtain for $L=1,2,3, \ldots$

$$
\left(\begin{array}{l}
B_{0}^{(L)}(z, q) \\
B_{1 / 2}^{(L)}(z, q)
\end{array}\right)=M^{(L)}(z, q)\left(\begin{array}{l}
B_{0}^{(L-1)}(z, q) \\
B_{1 / 2}^{(L-1)}(z, q)
\end{array}\right)
$$

where

$$
\begin{aligned}
M^{(L)}(z, q) & =\left(\begin{array}{cc}
1 & q^{L / 2} \\
q^{L / 2} & 1
\end{array}\right) & & \text { if } L \text { is even } \\
& =\left(\begin{array}{cc}
1 & z q^{(L+1) / 2} \\
z^{-1} q^{(L-1) / 2} & 1
\end{array}\right) & & \text { if } L \text { is odd. }
\end{aligned}
$$

Together with the initial conditions $B_{j}^{(0)}(z, q)=1$, these recursion relations fully determine the $B_{j}^{(L)}(z, q)$ for all $L$. On the other hand, the recursion relations satisfied by the $C_{j}^{(L)}$ are obtained by explicitly performing the summation over the variable $h_{L}$ on the rhs of (2.10). Omitting the (elementary) details, we find this way that the $C_{j}^{(L)}(z, q)$ are determined by precisely the same recursion relations and initial conditions as the $B_{j}^{(L)}(z, q)$, thus completing the proof.

To conclude this section, let us note that the polynomials of theorem (2.11) are reciprocal to the Rogers-Szegö polynomials (see e.g. [14]),

$$
H_{n}(z ; q)=\sum_{k=0}^{n} z^{k}\left[\begin{array}{l}
n \\
k
\end{array}\right]_{q}
$$

in the following sense:

$$
B_{j}^{(L)}\left(z^{-1}, q^{-1}\right)= \begin{cases}z^{-\left[\frac{L}{2}\right]} q^{-\left[\frac{L}{2}\right]\left[\frac{L+1}{2}\right]} H_{L}(z ; q) & \text { if } L \equiv 2 j(\bmod 2) \\ z^{-\left[\frac{L+1}{2}\right]} q^{-\left[\frac{L+1}{2}\right]\left[\frac{L+2}{2}\right]} H_{L}(z q ; q) & \text { if } L \equiv 2 j+1(\bmod 2)\end{cases}
$$

(The derivation of (2.16), starting from the definition (2.7), is elementary.) 


\section{Connection with the Heisenberg magnet}

Here we describe how the polynomials of the previous section arise (modulo a conjecture) in the one-dimensional Heisenberg model, also called the spin- $\frac{1}{2}$ XXX chain. The hamiltonian of this model is given by the hermitean operator

$$
H=J \sum_{n=1}^{L}\left(\mathbf{S}_{n} \cdot \mathbf{S}_{n+1}-\frac{1}{4}\right) \quad\left(\mathbf{S}_{L+1}=\mathbf{S}_{1}, J \in \mathbb{R}_{\neq 0}\right),
$$

acting on $\left(\mathbb{C}^{2}\right)^{\otimes L}$, where the components of $2 \mathbf{S}_{n}$ are the three Pauli matrices acting on the space $\mathbb{C}^{2}$ associated with the $n$-th site along the chain. We will summarize only the details of the solution of the model which are relevant for us here, following [15], and refer the reader to this reference for a more thorough discussion which also explains the terminology used below.

The diagonalization of the hamiltonian, using Bethe's Ansatz [13], leads to the following characterization of its eigenstates. First of all, one exploits the $S U(2)$ symmetry of the model, i.e. the fact that $H$ commutes with the total spin operator $\mathbf{S}=\sum_{n=1}^{L} \mathbf{S}_{n}$, to diagonalize it simultaneously with $\mathbf{S}^{2}$ (whose eigenvalues are $S(S+1)$ ) and $S^{z}$. Thus the spectrum splits into $s u(2)$ multiplets, and each $s u(2)$ highest-weight eigenstate with $M$ down-spins and $L-M$ up-spins (where $M=0,1, \ldots,\left[\frac{L}{2}\right]$ ), namely such that $S=S^{z}=\frac{1}{2}(L-2 M) \geq 0$, is labeled by a set of half-integers $\left\{\left\{I_{j}^{a}\right\}_{j=1}^{m_{a}}\right\}_{a=1}^{\infty}$, where the $m_{a}$ satisfy

$$
\sum_{a=1}^{\infty} a m_{a}=M=\frac{L}{2}-S
$$

(The non-negative integers $m_{a}$ are the number of "strings of length $a$ ", $a=1,2,3, \ldots$, in the given state.) For a given $a$, the $I_{j}^{a}$ are distinct integers (half-odd-integers) if $L-m_{a}$ is odd (even), lying in ranges which depend on the $m_{a}$ and the size of the system $L$ :

$$
\left|I_{j}^{a}\right| \leq \frac{1}{2}\left(L-\sum_{b=1}^{\infty} t_{a b} m_{b}-1\right)
$$

where $t_{a b}=2 \min (a, b)-\delta_{a b}$. Note that $\min (a, b)=\left(C_{T_{n}}^{-1}\right)_{a b}$ for all $a, b \leq n$, where $C_{T_{n}}$ is the Cartan matrix of the tadpole graph of $n$ nodes, i.e. the same as the Cartan matrix of the algebra $A_{n}$ except for the last entry along the diagonal which is $\left(C_{T_{n}}\right)_{n n}=1$. Hence the fact that $m_{a}=0$ for all $a>M=\frac{L}{2}-S$, implied by (3.2), allows us to rewrite (3.3) as

$$
\left|I_{j}^{a}\right| \leq \frac{1}{2}\left(L-\left(\mathbf{m}\left(2 C_{T_{M}}^{-1}-1\right)\right)_{a}-1\right) \quad(a=1,2, \ldots, M),
$$


where $\mathbf{m}=\left(m_{1}, m_{2}, \ldots, m_{M}\right)$ and 1 is the $M \times M$ identity matrix.

Now the hamiltonian (3.1) is translational invariant, and the eigenstate labeled by $\left\{I_{j}^{a}\right\}$ is also an eigenstate of the momentum (shift) operator with the eigenvalue

$$
P \equiv \frac{2 \pi}{L} \sum_{a=1}^{\infty} \sum_{j=1}^{m_{a}}\left(I_{j}^{a}+\frac{L}{2}\right) \quad(\bmod 2 \pi)
$$

This formula can be interpreted as saying that the $j$-th $a$-string in the state contributes

$$
p_{j}^{a}=\frac{2 \pi}{L}\left(I_{j}^{a}+\frac{L}{2}\right)
$$

to the total momentum $P$. (In fact, $p_{j}^{a}$ can be seen to be the sum of the $a$ "quasimomenta" 15 comprising the $j$-th $a$-string, provided - by convention - that the real parts of the quasi-momenta lie in the Brillouin zone $[0,2 \pi]$.) It follows from (3.4) that the momenta $p_{j}^{a}$ belong to the set $\left\{p_{\min }^{a}(\mathbf{m}), p_{\min }^{a}(\mathbf{m})+\frac{2 \pi}{L}, p_{\min }^{a}(\mathbf{m})+\frac{4 \pi}{L}, \ldots, p_{\max }^{a}(\mathbf{m})\right\}$, where

$$
p_{\min }^{a}(\mathbf{m})=\frac{\pi}{L}\left(\left(\mathbf{m}\left(2 C_{T_{M}}^{-1}-1\right)\right)_{a}+1\right)=2 \pi-p_{\max }^{a}(\mathbf{m}),
$$

and for all $a$ obey a fermionic exclusion rule

$$
p_{j}^{a} \neq p_{k}^{a} \quad \text { for } \quad j \neq k
$$

Let us now form the generating function of the total momentum $P$ in the sector $S=S^{z}=\frac{L}{2}-M$, ignoring the $\bmod 2 \pi$ in (3.5), namely consider (for $L$ a positive integer and $S \in\left\{0, \frac{1}{2}, 1, \ldots, \frac{L}{2}\right\}$ such that $\left.2 S \equiv L(\bmod 2)\right)$ the sum

$$
\mathcal{F}_{S}^{(L)}(q)=q^{-M} \sum_{\left\{p_{j}^{a}\right\}} q^{(L / 2 \pi) \sum_{a=1}^{\infty} \sum_{j=1}^{m_{a}} p_{j}^{a}}
$$

The summation here is performed over all sets $\left\{p_{j}^{a}\right\}$ allowed by (3.7)-(3.8) and (3.2), and the prefactor $q^{-M}$ was introduced for later convenience. Using the methods developed in [5] this sum is recast in the fermionic form

$$
\mathcal{F}_{S}^{(L)}(q)=q^{-M} \sum_{\substack{m_{a} \in \mathbb{Z} \\
\Sigma_{a=1}^{M} a m_{a}=M}} q^{\mathbf{m} C_{T_{M}}^{-1} \mathbf{m}^{t}} \prod_{b=1}^{M}\left[\begin{array}{c}
L-\left(\mathbf{m}\left(2 C_{T_{M}}^{-1}-1\right)\right)_{b} \\
m_{b}
\end{array}\right]_{q}
$$

where $M=\frac{L}{2}-S$. When $M=0$, eq. (邽) should be understood as $\mathcal{F}_{L / 2}^{(L)}(q)=1$. 
Conjecture: For all $L \in \mathbb{Z}$ and $S \in \frac{L}{2}+\mathbb{Z}$

$$
\mathcal{F}_{S}^{(L)}(q)=\mathcal{B}_{S}^{(L)}(q) \equiv\left[\begin{array}{c}
L \\
\frac{L}{2}-S
\end{array}\right]_{q}-q^{2 S+1}\left[\begin{array}{c}
L \\
\frac{L}{2}-S-1
\end{array}\right]_{q} .
$$

Note that the rhs here serves as the definition of $\mathcal{B}_{S}^{(L)}(q)$.

We have verified that (3.11) holds for many small values of $S$ and $L$; its validity at $q=1$, where the rhs reduces to $\left(\begin{array}{c}L \\ \frac{L}{2}-S\end{array}\right)-\left(\begin{array}{c}L \\ \frac{L}{2}-S-1\end{array}\right)$ (cf. (2.6)) which correctly counts all $s u(2)$ highest-weight states of spin $S$ in the spin chain, was proved in appendix A of [15].

The significance of the conjecture, in connection with the discussion of sect. 2, is revealed by noting the following relation. Starting from (2.7) we have for $L \equiv 2 j(\bmod 2)$

$$
\begin{aligned}
\left(z^{2} q\right)^{j / 2} B_{j}^{(L)}(z, q) & =\sum_{k \in j+\mathbb{Z}} z^{k} q^{k^{2}}\left[\begin{array}{c}
L \\
\frac{L}{2}+k
\end{array}\right]_{q} \\
& =\delta_{j, 0}\left[\begin{array}{c}
L \\
\frac{L}{2}
\end{array}\right]_{q}+\sum_{S \in j+\mathbb{Z} \geq 0}\left(z^{S}+z^{-S}\right) q^{S^{2}}\left[\begin{array}{c}
L \\
\frac{L}{2}-S
\end{array}\right]_{q} \\
& =\delta_{j, 0}\left[\begin{array}{c}
L \\
\frac{L}{2}
\end{array}\right]_{q}+\sum_{S \in j+\mathbb{Z} \geq 0}\left([2 S+1]_{z}-[2 S-1]_{z}\right) q^{S^{2}}\left[\begin{array}{c}
L \\
\frac{L}{2}-S
\end{array}\right]_{q} \\
& =\sum_{S \in j+\mathbb{Z}_{\geq 0}}[2 S+1]_{z} q^{S^{2}} \mathcal{B}_{S}^{(L)}(q),
\end{aligned}
$$

where $[x]_{z}=\left(z^{x / 2}-z^{-x / 2}\right) /\left(z^{1 / 2}-z^{-1 / 2}\right)$ (so that $[2 S+1]_{z}$ is the character $\operatorname{Tr} z^{S^{z}}$ of the spin- $S$ unitary representation of the algebra $s u(2))$.

It is now natural to extend the generating functions (3.9) to the full spectrum of the hamiltonian, consisting of whole $s u(2)$ multiplets, by forming the fermionic sums

$$
\begin{aligned}
\hat{F}_{j}^{(L)}(z, q)= & \left(z^{2} q\right)^{-j / 2} \sum_{S \in j+\mathbb{Z} \geq 0}[2 S+1]_{z} q^{S^{2} \mathcal{F}_{S}^{(L)}(q)} \\
= & \left(z^{2} q\right)^{-j / 2} \sum_{S=j}^{L / 2}[2 S+1]_{z} q^{S(S+1)-L / 2} \\
& \times \sum_{\substack{m_{a} \in \mathbb{Z} \\
\Sigma_{a=1}^{\infty} a m_{a}=L / 2-S}} q^{\mathbf{m} C_{T_{\infty}}^{-1} \mathbf{m}^{t}} \prod_{b=1}^{\infty}\left[\begin{array}{c}
L-\left(\mathbf{m}\left(2 C_{T_{\infty}}^{-1}-1\right)\right)_{b} \\
m_{b}
\end{array}\right]_{q},
\end{aligned}
$$

where $j=0$ or $\frac{1}{2}, L \equiv 2 j(\bmod 2)$, and the sum over $S$ is in steps of 1 . (Here $C_{T_{\infty}}^{-1}$ is to be understood as the infinite matrix whose elements are $\min (a, b)$; all the sums and 
products in (3.13) are nevertheless finite, as automatically enforced by the restriction (3.2).) Assuming (3.11) holds, we conclude from (3.12) that the sums $\hat{F}_{j}^{(L)}(z, q)$ provide another representation for the polynomials of theorem (2.11). The evaluation of these polynomials at $z=q=1$ is most easily performed using the representation (2.9), which immediately gives $C_{j}^{(L)}(1,1)=2^{L}$. From the point of view of the spin- $\frac{1}{2}$ Heisenberg chain the fact

that $\hat{F}_{j}^{(L)}(1,1)=2^{L}$ demonstrates 13 15] 16 the completeness of the Bethe-Ansatz eigenstates - taking into account also the $S U(2)$ symmetry of the model - for a finite chain of $L$ sites.

We conclude this section by making two comments. First, it is possible to convert the expressions in eqs. (3.10) and (3.13) into a form which suggests a relation to the fermionic sum representations [6] 9 for the (finitized) characters of the unitary minimal models. To do so we make the variable change $m_{a} \mapsto m_{a}^{\prime}=L-2 \sum_{b=1}^{M} m_{b}\left(C_{T_{M}}^{-1}\right)_{b, M+1-a}$ in (3.10), which leads to

$$
\begin{gathered}
\mathcal{F}_{S}^{(L)}(q)=q^{\frac{L}{2}\left(\frac{L}{2}-1\right)-S(S-1)} \sum_{m_{2}^{\prime}, \ldots, m_{M}^{\prime} \in 2(\mathbb{Z}+S)} q^{\frac{1}{4} \mathbf{m}^{\prime} C_{A_{M}} \mathbf{m}^{\prime t}-\frac{1}{2} L m_{M}^{\prime}} \\
\times \prod_{b=1}^{M}\left[\begin{array}{c}
\frac{1}{2}\left(\left(\mathbf{m}^{\prime} I_{A_{M}}\right)_{b}+m_{1}^{\prime} \delta_{b, 1}+L \delta_{b, M}\right) \\
m_{b}^{\prime}
\end{array}\right]_{q}
\end{gathered}
$$

where $M=\frac{L}{2}-S$ as before, $m_{1}^{\prime}=2 S$ (which follows from (3.2)), and $C_{A_{M}}\left(I_{A_{M}}\right)$ is the Cartan (incidence) matrix of the algebra $A_{M}$. Similarly, eq. (3.13) can be rewritten as

$$
\begin{aligned}
& \hat{F}_{j}^{(L)}(z, q)=\left(z^{2} q\right)^{-j / 2} q^{\frac{L}{2}\left(\frac{L}{2}-1\right)} \sum_{m_{1}^{\prime}, \ldots, m_{M}^{\prime} \in 2(\mathbb{Z}+j)}\left[m_{1}^{\prime}+1\right]_{z} \\
& \times q^{\frac{1}{4} \mathbf{m}^{\prime} C_{A_{M}} \mathbf{m}^{\prime t}+\frac{1}{2}\left(m_{1}^{\prime}-L m_{M}^{\prime}\right)} \prod_{b=1}^{M}\left[\begin{array}{c}
\left.\frac{1}{2}\left(\left(\mathbf{m}^{\prime} I_{A_{M}}\right)_{b}+m_{1}^{\prime} \delta_{b, 1}+L \delta_{b, M}\right)\right]_{q} \\
m_{b}^{\prime}
\end{array},\right.
\end{aligned}
$$

where $M=\left(L-m_{1}^{\prime}\right) / 2$.

Second, recall that the sum (3.13) originated from the quasiparticle sum (3.9) which - potentially, as $L \rightarrow \infty$ - involves infinitely many types of quasiparticles $a=1,2,3, \ldots$ We contrast it here with the 2-quasiparticle sum (2.8), explicitly exhibiting the latter's quasiparticle interpretation. As in [5], we can rewrite (2.8) as

$$
F_{j}^{(L)}(z, q)=\sum_{m_{ \pm}=0}^{\infty} z^{m_{+}-m_{-}} \sum_{\left\{p_{j}^{ \pm}\right\}_{j=1}^{m_{ \pm}}} q^{(L / 2 \pi) \sum_{a= \pm} \sum_{j=1}^{m_{a}} p_{j}^{a}}
$$


where $p_{j}^{a} \in\left\{p_{\min }^{a}\left(m_{ \pm}\right), p_{\min }^{a}\left(m_{ \pm}\right)+\frac{2 \pi}{L}, \ldots, p_{\max }^{a}\left(m_{ \pm}\right)\right\}, p_{j}^{a} \neq p_{k}^{a}$ for $j \neq k$, and

$$
\begin{aligned}
& p_{\min }^{+}\left(m_{ \pm}\right)=\frac{\pi}{L}\left(m_{+}-m_{-}+4 j+1\right)=p_{\max }^{+}\left(m_{ \pm}\right)-\frac{2 \pi}{L}\left[\frac{L-1}{2}-j\right] \\
& p_{\min }^{-}\left(m_{ \pm}\right)=\frac{\pi}{L}\left(m_{-}-m_{+}-4 j+1\right)=p_{\max }^{-}\left(m_{ \pm}\right)-\frac{2 \pi}{L}\left(\left[\frac{L}{2}+j\right]-1\right)
\end{aligned}
$$

\section{Characters}

In the limit $L \rightarrow \infty$ the polynomials discussed in the previous sections become infinite series. We will use the symbols $C_{j}^{(\infty)}(z, q), \mathcal{F}_{S}^{(\infty)}(q)$, and $\hat{F}_{j}^{(\infty)}(z, q)$ to denote the $L \rightarrow \infty$ limits of the rhs's of eqs. (2.10), (3.10), and (3.13), respectively. In the case of the $B_{j}^{(L)}(z, q), F_{j}^{(L)}(z, q)$, and $\mathcal{B}_{S}^{(L)}(q)$ the following simplifications occur due to (2.5):

$$
\begin{gathered}
B_{j}^{(\infty)}(z, q)=\frac{1}{(q)_{\infty}} \sum_{k \in \mathbb{Z}} z^{k} q^{k(k+2 j)}, \quad \mathcal{B}_{S}^{(\infty)}(q)=\frac{1-q^{2 S+1}}{(q)_{\infty}}, \\
F_{j}^{(\infty)}(z, q)=\sum_{m_{1}, m_{2}=0}^{\infty} z^{m_{1}-m_{2}} \frac{q^{\frac{1}{2} \mathbf{m} C_{A_{2}} \mathbf{m}^{t}+2 j\left(m_{1}-m_{2}\right)}}{(q)_{m_{1}}(q)_{m_{2}}} .
\end{gathered}
$$

The series in (4.1) are familiar expressions for characters of two important infinitedimensional Lie algebras: $B_{j}^{(\infty)}(z, q)$ with $j=0, \frac{1}{2}$ are [3] [17] the (normalized) characters $\chi_{j}=\operatorname{Tr} z^{J_{0}^{z}-j} q^{L_{0}-j / 4}$ of the two integrable highest-weight representations of $\left(A_{1}^{(1)}\right)_{1}$, the $s u(2)$ affine Kac-Moody algebra at level 1 ; the $\mathcal{B}_{S}^{(\infty)}(q)$, on the other hand, are (see e.g. [2]) the characters $\chi_{S}^{\mathrm{Vir}}=\operatorname{Tr} q^{L_{0}-S^{2}}$ of the irreducible highest-weight representations of the Virasoro algebra at central charge $c=1$ and highest-weight $\Delta=S^{2}, \quad S \in \frac{1}{2} \mathbb{Z}_{\geq 0}$. The well-known expansion

$$
\left(z^{2} q\right)^{j / 2} \chi_{j}(z, q)=\sum_{S \in j+\mathbb{Z} \geq 0}[2 S+1]_{z} q^{S^{2}} \chi_{S}^{\mathrm{Vir}}(q)
$$

showing the decomposition of the $\left(A_{1}^{(1)}\right)_{1}$ representations into (degenerate) Virasoro representations, can be recovered from eq. (3.12) in the limit $L \rightarrow \infty$.

Thus we have

Corollary (of theorem (2.11)):

$$
\chi_{j}(z, q)=F_{j}^{(\infty)}(z, q)=C_{j}^{(\infty)}(z, q) \quad\left(j=0, \frac{1}{2}\right) .
$$


In addition, validity of the conjecture (3.11) implies in the limit $L \rightarrow \infty$

$$
\chi_{S}^{\operatorname{Vir}}(q)=\mathcal{F}_{S}^{(\infty)}(q) \quad\left(S=0, \frac{1}{2}, 1, \ldots\right)
$$

and if (4.5) is true, eq. (4.3) yields the identity

$$
\chi_{j}(z, q)=\hat{F}_{j}^{(\infty)}(z, q) \quad\left(j=0, \frac{1}{2}\right)
$$

The rhs of eq. (4.6) was constructed in sect. 3 from the Bethe-Ansatz data of the spin- $\frac{1}{2}$ XXX chain. Its equality to the characters of $\left(A_{1}^{(1)}\right)_{1}$ provides further support to the observation [12] that the long-distance asymptotics of the antiferromagnetic chain is described by the level one $S U(2)$ WZW model (perturbed by some marginal operators [18]). The work of [12] is concerned mainly with the symmetry of the model in the continuum limit, while (4.6) is a statement about the degeneracy structure of the spectrum.

\section{Discussion}

Several comments on eqs. (4.4)-(4.6) are in order. The first equality in (4.4) (specialized to $z=1$ ) has recently appeared in [19], where a new construction of highest-weight representations of certain subalgebras of affine Lie algebras was presented. In particular, representations of $A_{1}^{(1)}$ were obtained there by considering a subalgebra of $A_{2}^{(1)}$ which is isomorphic to $A_{1}^{(1)}$ (this "explains" the appearance of the Cartan matrix of $A_{2}$ in (4.2)).

The equality $\chi_{j}(z, q)=F_{j}^{(\infty)}(z, q)$ can easily be inferred also from the results of [8]. In the latter work fermionic expressions of the type (4.2) were given for the theta functions

$$
f_{a, b}(z, q)=\frac{1}{(q)_{\infty}} \sum_{k \in \mathbb{Z}} z^{k+\frac{b}{2 a}} q^{a\left(k+\frac{b}{2 a}\right)^{2}} \quad\left(a \in \mathbb{Z}_{>0}, \quad b \in \mathbb{Z}\right)
$$

Taking $a=1$ and $b=2 j=0,1$, where $f_{1,2 j}(z, q)=\left(z^{2} q\right)^{j / 2} B_{j}^{(\infty)}(z, q)$, they yield the representation

$$
\chi_{j}(z, q)=\sum_{\substack{m_{1}, m_{2}=0 \\ m_{1}-m_{2} \equiv 2 j(\bmod 2)}}^{\infty} z^{\frac{m_{1}-m_{2}}{2}-j} \frac{q^{\left(\frac{m_{1}+m_{2}}{2}\right)^{2}-j^{2}}}{(q)_{m_{1}}(q)_{m_{2}}}
$$

for the $\left(A_{1}^{(1)}\right)_{1}$ characters, which is slightly different from (4.2). However, if the expressions given in $[8]$ for the $f_{4, b}(z, q)$ are inserted into the rhs of the simple identity $f_{1,2 j}(z, q)=$ $f_{4,4 j}\left(z^{2}, q\right)+f_{4,4(j+1)}\left(z^{2}, q\right)$, then (4.2) is obtained. 
The main difference between the 2-quasiparticle sums (4.2) and (5.2) is in the quadratic form in the exponent of $q$. In fact, there exists an infinite family of such sums with different quadratic forms. Their finitized version is obtained by considering the following generalization of $B_{j}^{(L)}(z, q)$,

$$
B_{j}^{(L ; N)}(z, q)=\sum_{k \in \mathbb{Z}} z^{k} q^{k(k+2 j)}\left[\begin{array}{c}
L \\
{\left[\frac{L}{2}+j\right]+N k}
\end{array}\right]_{q} \quad(N=1,2,3, \ldots)
$$

which reduces to (2.7) when $N=1$. Performing essentially the same manipulations as in part (i) of the proof of theorem (2.11) leads to the identity $B_{j}^{(L ; N)}(z, q)=F_{j}^{(L ; N)}(z, q)$, where

$$
\begin{aligned}
F_{j}^{(L ; N)}(z, q) & =\sum_{\substack{m_{1}, m_{2} \in \mathbb{Z} \\
m_{1}-m_{2} \equiv(\bmod N)}} z^{\frac{m_{1}-m_{2}}{N}} q^{\frac{1}{2} \mathbf{m} B_{N} \mathbf{m}^{t}+\frac{2 j}{N}\left(m_{1}-m_{2}\right)}\left[\begin{array}{c}
\left.\frac{L+1}{2}-j\right] \\
m_{1}
\end{array}\right]_{q}\left[\begin{array}{c}
\left.\frac{L}{2}+j\right] \\
m_{2}
\end{array}\right]_{q} .
\end{aligned}
$$

Here $B_{N}=\left(\begin{array}{cc}2 / N^{2} & 1-2 / N^{2} \\ 1-2 / N^{2} & 2 / N^{2}\end{array}\right)$, and so $B_{1}=C_{A_{2}}$ while $\frac{1}{2} \mathbf{m} B_{2} \mathbf{m}^{t}=\left(\frac{m_{1}+m_{2}}{2}\right)^{2}$, corresponding to (4.2) and (5.2), respectively. Now in the infinite $L$ limit we have $\chi_{j}(z, q)=B_{j}^{(\infty ; N)}(z, q)=F_{j}^{(\infty ; N)}(z, q)$ independently of $N$. But at finite $L$ the sums (5.3)-(5.4) do depend on $N$. In particular, their value at $z=q=1$ is easily calculable when $L \equiv 0(\bmod 2 N)$ :

$$
\begin{aligned}
B_{j}^{(L ; N)}(1,1) & =\sum_{\substack{k=0 \\
k \equiv 0 \text { (mod } N)}}\left(\begin{array}{l}
L \\
k
\end{array}\right) \\
& =\frac{1}{N} \sum_{j=0}^{N-1}\left(2 \cos \frac{j \pi}{N}\right)^{L}=\frac{1}{2 N} \operatorname{Tr}\left(I_{A_{2 N-1}^{(1)}}\right)^{L}
\end{aligned}
$$

where $I_{A_{n}^{(1)}}$ is the incidence matrix of the Dynkin diagram of the affine Lie algebra $A_{n}^{(1)}$.

The most rhs of eq. (5.5) is naturally interpreted as the total number of states in a "spin chain" of $L$ sites with periodic boundary conditions, where the states (or heights) $h_{\ell} \in\{0,1, \ldots, 2 N-1\}$ at each site $\ell=1,2, \ldots, L$ are labeled by the nodes of the Dynkin diagram of $A_{2 N-1}^{(1)}$ whose incidence matrix imposes the adjacency constraint $h_{\ell+1}-h_{\ell} \equiv$ $\pm 1(\bmod 2 N)$, and furthermore one of the heights is fixed (say $\left.h_{1}=0\right)$. This suggests a connection between the polynomials (5.3)-(5.4) and the spectrum of the hamiltonian obtained from the transfer matrix of the critical RSOS lattice model based on $A_{2 N-1}^{(1)}$ [20]. 
The conformal field theory associated with the latter model is identified [20] as the $c=1$ gaussian model at compactification radius $r=\frac{N}{\sqrt{2}}$, which is [2] a $\mathbb{Z}_{N}$ orbifold of the level one $S U(2)$ WZW model. This orbifold relation allows the construction of the affine characters $\left(z^{2} q\right)^{j / 2} \chi_{j}(z, q)=f_{1,2 j}(z, q)$ as the sums $\sum_{b=1}^{N} f_{N^{2}, 2(b+j) N}\left(z^{N}, q\right)$ of characters of the $r=\frac{N}{\sqrt{2}}$ gaussian model. The fact that the affine characters $\chi_{j}$ are recovered in the $L \rightarrow \infty$ limit of (5.3)-(5.4) is therefore consistent with the connection suggested above.

Next, the identity $\chi_{0}(1, q)=C_{0}^{(\infty)}(1, q)$, which is a special case of the second equality in (4.4), was first shown in [21], where an explicit construction of the vacuum representation of $\left(A_{n}^{(1)}\right)_{1}$ on a space of paths in $\mathbb{Z}_{n+1}$ is presented. This construction was generalized to higher levels and arbitrary integrable representations in [22]. As far as we know, the conjectures (4.5)-(4.6) are new.

Returning to sect. 2, it would be interesting to generalize theorem (2.11) to polynomial identities between different forms of finitized characters of representations of higher rank and level affine Lie algebras. The relevant fermionic and 1D configuration sum expressions for the characters themselves are known in various cases [19]-[22]. The connection between the finitized characters and the spectrum of finite spin chains, discussed in sect. 3 for the case of $\left(A_{1}^{(1)}\right)_{1}$, should also be further explored and generalized. This may improve our understanding of the scaling limits of spin chains which are relevant to quantum field theory.

Note added. I have been recently informed by A.N. Kirillov that he has a proof [23] of conjecture (3.11) (and hence also of eq. (4.5)).

Acknowledgements. I would like to thank A. Berkovich, F. Eßler, V. Korepin and B. McCoy for useful discussions. This work was supported in part by the NSF, grant 91-08054, and by the US-Israel Binational Science Foundation. 


\section{References}

[1] A.A. Belavin, A.M. Polyakov and A.B. Zamolodchikov, Nucl. Phys. B241 (1984) 333.

[2] P. Ginsparg, in: Fields, strings, and critical phenomena, Les Houches 1988, ed. E. Brézin and J. Zinn-Justin (North Holland, Amsterdam, 1989).

[3] V.G. Kac, Infinite dimensional Lie algebras, third edition (Cambridge University Press, 1990).

[4] R.J. Baxter, Exactly solved models in statistical mechanics (Academic Press, London, 1982).

[5] R. Kedem and B.M. McCoy, J. Stat. Phys. 71 (1993) 865 (hep-th/9210129); S. Dasmahapatra, R. Kedem, B.M. McCoy and E. Melzer, Stony Brook preprint, hepth/9304150, J. Stat. Phys. (in press).

[6] R. Kedem, T.R. Klassen, B.M. McCoy and E. Melzer, Phys. Lett. B304 (1993) 263 and B307 (1993) 68 (hep-th/9211102 and 9301046).

[7] M. Terhoeven, Bonn preprint, hep-th/9111120; A. Kuniba, T. Nakanishi, J. Suzuki, Mod. Phys. Lett. A8 (1993) 1649 (hep-th/9301018).

[8] R. Kedem, B.M. McCoy and E. Melzer, Stony Brook preprint, hep-th/9304056, to appear in C.N. Yang's 70th birthday Festschrift, ed. S.T. Yau.

[9] E. Melzer, Stony Brook preprint, hep-th/9305114, Int. J. Mod. Phys. A (in press).

[10] G.E. Andrews, R.J. Baxter and P.J. Forrester, J. Stat. Phys. 35 (1984) 193.

[11] A. Rocha-Caridi, in: Vertex operators in mathematics and physics, ed. J. Lepowsky, S. Mandelstam and I.M. Singer (Springer, Berlin, 1985).

[12] I. Affleck, Phys. Rev. Lett. 55 (1985) 1355.

[13] H. Bethe, Z. Phys. 71 (1931) 205.

[14] G.E. Andrews, The theory of partitions (Addison-Wesley, London, 1976).

[15] M. Takahashi, Progr. Theor. Phys. 46 (1971) 401.

[16] L.D. Faddeev and L.A. Takhtajan, Phys. Lett. A85 (1981) 375.

[17] A.J. Feingold and J. Lepowsky, Adv. in Math. 29 (1978) 271.

[18] I. Affleck, D. Gepner, H.J. Schulz and T. Ziman, J. Phys. A22 (1989) 511.

[19] B.L. Feigin and A.V. Stoyanovsky, preprint, hep-th/9308079.

[20] V. Pasquier, J. Phys. A20 (1987) L1229.

[21] E. Date, M. Jimbo, A. Kuniba, T. Miwa and M. Okado, Lett. Math. Phys. 17 (1989) 51.

[22] M. Jimbo, K.C. Misra, T. Miwa and M. Okado, Commun. Math. Phys. 136 (1991) 543.

[23] A.N. Kirillov, private communication. 